component of the curriculum vitae. Evidence of increased interest in research is that in recent years more trainees have entered papers for the College Research Prize, although this remains a tiny number. Prior to the day, one of us (CB) had presented Dr Creed with a list of problems encountered by trainees attempting to do research, gathered from trainees at trainees' days held throughout the UK. A number of problems and possible solutions were discussed:

(a) no consultant help! - find another consultant who will help, or choose a post on your rotation scheme with the prime aim of working for a consultant who is known to supervise trainee's research

(b) there is an emphasis on large projects and publications! - not true, small projects are just as likely to get completed, possibly published and certainly to bring credit to the trainee

(c) unable to get funding! - only a minority of trainees get on to funded projects to begin with, smaller projects can demonstrate one's ability to complete and may lead to bigger projects

(d) lack of research training! - get on a course run by the college Research Committee which is particularly aimed at trainees from non-teaching centres.

Dr Creed then presented an autobiographical account of his development as a researcher as encouragement to trainees who had experience of failure in their early projects. He gave an account of small projects that could be completed within a six month placement. We were given a brief handout to illustrate how an idea can be turned into a feasible project; this exercise stimulated much enthusiasm, and encouraged trainees to get started with a project.

Trainees then divided into three groups to discuss: trainees doing research; psychotherapy training; and the implications for training arising from working in community settings. The group discussing psycho- therapy training reported a lack of availability of training opportunities across the psychotherapies, with some trainees experiencing severe difficulties and occasionally obstructive attitudes. A need was perceived for a more formal approach to training, appropriate to the level of each trainee, with the recognition that a trainee may have a flair for, e.g., behavioural psychotherapy rather than psychodynamic psychotherapy or vice versa. The group looking at trainees and research recognised an element of "distasteful self advancement" in the need to do research but also the benefits to personal intellectual satisfaction and the contribution research makes to the development of clinical services. A common difficulty was lack of supervision and it was felt that if the local tutor was not a "clinical researcher" they should direct trainees to someone able and willing to offer supervision. It was pointed out that at each College Quarterly Meeting there is an opportunity for trainees to present short papers. The group addressing the training implications of working in community settings identified a number of potential obstacles to training that need to be overcome. Concern was expressed about difficulties posed by travelling, the likely loss of mutual support and learning from being part of a peer group. Trainees felt uncertain about what the future holds in store, bearing in mind that community resources often appear to be underfunded and understaffed.

Judging by comments from trainees, the day proved informative and a feeling of shared interests developed. We thank the guest speakers for their helpful and stimulating contributions to the day. The next trainees' day of the North East Division will be held in Newcastle. As always, all trainees including those who are not inceptors will be welcome to attend.

\title{
The report of a working party of the fourth Collegiate Trainees' Committee
}

\author{
The public image of psychiatry - a pilot study
}

\author{
Dinesh BHUGRa Senior Registrar, The Maudsley Hospital, Denmark Hill, London \\ SE5 8AZ (correspondence); and JAN ScoTT Lecturer (now Senior Lecturer) in \\ Psychiatry, University of Newcastle-upon-Tyne
}

Attitudes towards psychiatry are likely to affect psychiatrists and their patients in a number of ways. The success of the move towards community based services is directly dependent upon the public's attitudes 
to the mentally ill living among them. Political and social attitudes are likely to affect the funding of these services as well as recruitment into these services. This was the primary reason why the fourth Collegiate Trainees' Committee (CTC) decided to look at the attitudes towards mental illness.

There have been few studies in the UK looking at the attitudes and the possible attributional factors for these attitudes. Nierdzik \& Cochrane (1985) showed that public attitudes towards the mentally ill were generally rejecting and that for the more severely behaviourally disturbed patients, the greater was the social rejection. With several American studies looking at the attitudes and the effects of factors like age, sex, religion, and education, on these attitudes (Cumming \& Cumming, 1957, Brockman \& Darcy, 1978; Scott et al, 1983) it was decided to incorporate some of their questions into the questionnaire for the present study. With the discharge of long-term patients into the community, the social rejection of the mentally disordered takes on a new dimension. Kirk \& Therrien (1975) point out that there is a wellestablished, although slowly changing, pattern of fear, anxiety and revulsion in response to the mentally ill. Any patient returning to the community thus faces these attitudes. These attitudes may reflect personal experiences and the effects of the media. Relatives often feel powerless and mystified and look to the expert for a satisfying explanation for these strange events (Ingham, 1985). With slow progress in the mental state, feelings of resentment may build up. Even though television is said to be a powerful medium (McLean, 1969) the psychiatrist is often caricatured and lampooned. Psychiatry has rarely been able to take advantage of the media as a platform to dispel fears and reduce ignorance about mental health.

The present study was intended as a pilot study to look at the public's attitudes towards mental illness.

\section{The study}

Members of the working party circulated the questionnaire (designed primarily by JS and approved by the working party) to GP surgery attenders. The largest subsample of 47 was from a single surgery attendance at a practice in Eastbourne. A total of 99 questionnaires were obtained. Of these, five were rejected because of a large number of incomplete responses. The sample is not totally representative of the general population but for such a pilot study can be considered adequate. Chi square analyses were carried out on the responses in the questionnaire. Copies of the questionnaire are available from the authors.

\section{Findings}

Of 94 respondents, 62 were female ( 33 married, 17 single, 8 divorced or separated and 3 widowed); 27 male (16 married, 8 single, 3 widowed) and 5 sex not known. Ten females were housewives and 34 were employed and 13 out of 27 males were employed.

Of the total respondents, 70 thought that more than $5 \%$ of the population see a psychiatrist. Sixteen respondents (17\%) agreed that mentally ill people were more violent than people in the general population and $58(62 \%)$ respondents disagreed. A large majority $77(82 \%)$ disagreed that mentally ill people are generally inadequate should pull themselves together and only $4(4 \%)$ agreed. Fifty-seven respondents $(61 \%)$ agreed that mental illness is like any other illness and more than a quarter (26 out of 94) disagreed. Again a majority, $59(63 \%)$, agreed that mental illness results from psychological or social problems and only $12(13 \%)$ disagreed although 17 $(18 \%)$ did not know. Eighteen (19\%) disagreed that mental illness is a disturbance of the nervous system and $30(32 \%)$ said that they did not know and only 39 $(41 \%)$ agreed.

When asked about mental hospitals, 17 (18\%) agreed that they seemed like prisons to them (of $44 \%$ disagreeing and $31 \%$ not knowing). An overwhelming majority, 80 (85\%), agreed that mental hospitals are necessary for the treatment of mentally ill (only four disagreeing) and $65(69 \%)$ disagreed that these should be situated away from towns. Ninety respondents $(96 \%)$ agreed that mental hospitals should be easy to reach for friends and family and the two respondents who disagreed were both males. Seventy-five respondents $(80 \%)$ disagreed that the mental hospitals should be surrounded by high fences and have guards, only, five agreed. Fifty-three respondents (56\%) agreed that most wards should be open in mental hospitals, 23 respondents did not know and five did not express any opinions. Only 12 respondents would specifically plan to be in to watch a programme on mental illness on TV and 52 would watch it if it came on. Twenty-one respondents would only watch it if other choices were of no interest to them and seven would turn off or change channels.

Of 94 respondents, 78 read a daily or Sunday paper and 12 did not. Only four respondents (three males) would object to the opening of a hostel for the mentally ill in their street. The commonest reason was the worry about the property prices falling.

Twenty-one respondents understood a psychiatrist to be a psychologist trained to treat mental illness whereas a majority $62(64 \%)$ recognised a psychiatrist as a doctor specialising in the treatment of mental illness. Thirty-five respondents suggested that one person in 1000 suffered from schizophrenia and 31 felt that the incidence of depression in the 
population was one in 1000 and 29 that one in 100 suffered from depression. Thirteen out of 94 (16 gave no response) said that psychiatrists in this country abuse their position. Fifty-nine out of 94 felt that some people are so ill they should stay in a mental hospital for prolonged periods. A majority of respondents (41 out of 72 (22 did not know)) disagreed that people with severe mental illness should be made to stay in hospital by law, even if it is against their wishes. More females agreed with the first preposition (about mentally ill staying for prolonged periods in hospitals) and females were less likely to be libertarian for the second preposition (about being detained against their wishes) even though nearly a quarter did not know. Out of 94 respondents, 77 would agree to see a psychiatrist if so recommended. Nearly a quarter of the respondents had had someone in their family suffer from psychiatric/ emotional/nervous or drink problems.

\section{Comment}

There was an overwhelming number of female respondents. This is useful in that it is the female who usually ends up looking after the sick in the family. But since this was not a random sample it is difficult to generalise from these findings. Females were more likely to confuse a psychiatrist and a psychologist which needs to be related to the educational attainment of the respondents - a factor not studied in the present sample.

Males were more likely to object to the opening of a hostel for the mentally ill in their street although the numbers are very small. This needs to be tested further with larger samples if hostility to the mentally ill is to be avoided. Males were also more likely to turn the TV off or change channels if a programme on mental illness came on. However a majority of female respondents would watch such a programme. As McLean found, television is an important source of education and psychiatry needs to learn to use this medium effectively in putting its views across.

When looking at the perceived characteristics of the mentally ill, nearly a fifth of the female respondents agreed that they were more violent. The females may be presenting their personal experiences or reflecting how the public views the mentally ill. Interestingly an overwhelming majority of males as well as females disagreed that the mentally ill were inadequate which would reflect experience of mental illness among family, friends and acquaintances.

Fifty-nine per cent of female respondents and $66 \%$ of male respondents saw mental illness like any other illness which is a higher figure than the $27 \%$ reported by Lyketsos et al (1985) among the visitors to a mental hospital. It is difficult to explain the discrepancy between the libertarian attitudes of the female respondents and their lower response. It may be worth looking at the individual's own definitions of illness and mental illness.

Seventy per cent of females and $62 \%$ males agreed that mental illness results from psychological or social problems, a figure similar to that of Lyketsos et al's 1985 study. However, in the present study the statement read psychological or social problems whereas Lyketsos's study had psychological and social problems. Less than half of females and one third of males saw mental illness as a disturbance of the nervous system, again similar figures to Lyketsos's work although they had not differentiated between responses according to sex. The respondents of both sexes had generally libertarian attitudes in their views of mental hospitals. That an overwhelming majority of male respondents and $77 \%$ of female respondents read a daily or Sunday paper suggests that access to the media can modify the opinions of the public and garner support. The public by a majority feels that mental hospitals are necessary for the treatment of the mentally ill and should be easily accessible.

There appears to be a small but palpable resistance to the mentally ill settling in the community, especially by the male respondents. There is some degree of confusion about the role of the psychiatrist and $14 \%$ of respondents see psychiatrists abusing their position. This may reflect a degree of fear and a lack of knowledge about mental illness among the respondents. Not only there is a need for education about mental illness, there is an indication for educating the community about the role of each member of the multidisciplinary team if community psychiatry is to succeed.

\section{Concluding remarks}

(a) Attitudes of the public to mental illness and psychiatry vary according to gender and other factors.

(b) The media can play an important role in education and gathering support for mentally ill and psychiatric programmes. Psychiatrists need to learn how to use the media properly.

(c) Mental hospitals are still seen as necessary for the treatment of the mentally ill and there is a slight resistance to the opening of community hostels for the mentally ill, especially among men.

(d) The attitudes of the present sample are libertarian but larger and tightly constructed samples need to be studied.

\section{Acknowledgements}

Thanks are due to all the respondents for their time and effort. The help of Mrs Jean Wales and Miss 
Sue Prevost at the Royal College of Psychiatrists is gratefully acknowledged.

\section{References}

Brockman, J. \& D'ARCY, C. (1978) Correlates of attitudinal social distance toward the mentally ill: a review and resurvey. Social Psychiatry, 13, 69-77.

Cumming, E. \& Cumming, J. (1957) Closed Ranks: An Experiment in Mental Health. Cambridge, Mass: Harvard University Press.

INGHAM, J. (1985) The public image of psychiatry. Social Psychiatry, 20, 107-108.

KIRK, S. \& THERRIEN, M. (1975) Community mental health myths and the fate of former hospitalised patients. Psychiatry, 38, 209-217.

Lyketsos, G., Mouyas, A., Mallion, M. et al (1985)

Opinion of public and patients about mental illness and psychiatric care in Greece. British Journal of Social and Clinical Psychology, 3, 59-66.

MCLEAN, U. (1969) Community attitudes to mental illness in Edinburgh. British Journal of Preventive and Social Medicine, 23, 45-52.

Nierdzik, K. \& Cochrane, R. (1985) Public attitudes towards mental illness - the effects of behaviour, role and psychiatric labels. International Journal of Social Psychiatry, 31, 23-33.

SCOTT, R., BlACH, P. \& FLYNN, T. (1983) A comparison of community attitudes toward CMHC services and clients with those of mental hospitals. American Journal of Community Psychology, 11, 741-749.

The members of the working party were Drs D. Bhugra (convenor), D. Cotteril, I. Davidson, S. McAuley, I. Medley, G. Mezey, C. Shapiro, J. Scott and P. White.

\section{Psychiatry abroad}

The Collegiate Trainees' Committee have formed a working party to look at psychiatry abroad and welcome information from any Members of the College who have experience of working as psychiatrists in Europe and the New Commonwealth, in particular about training requirements and standards in those countries where they have worked and any advice they could offer to anyone considering working abroad. All information would be dealt with in strict confidence. Please contact $\mathrm{Dr}$ Michael van Beinum, 87 Bruntsfield Place, Edinburgh, Scotland EH10 4HG

\section{Attendance at Quarterly Meetings}

Council has agreed that, in future, all Members must register if they wish to attend Quarterly Meetings. Although it has previously been College policy to enable Members to attend the Scientific Sessions without registering, the costs of hiring lecture theatres, audio-visuals and printing are now so high that Council has decided that Members attending should pay a greater contribution. Although the meetings will continue to be subsidised, the daily registration fee will be increased from the Annual General Meeting 1989.

April 1989

\section{Careers Fairs}

The Public Education Committee is taking over the College representation at Careers Fairs. At present, information packs are available but there are plans to provide a poster and audio-visual material for a stand which will better promote the image and opportunities of psychiatry. 